\title{
Linear motors based on piezoelectric MEMS
}

\author{
Víctor Ruiz-Díez ${ }^{1 *}$, Jorge Hernando-García ${ }^{1}$ and José Luis Sánchez-Rojas ${ }^{1}$ \\ 1 Microsystems, Actuators and Sensors Group, Universidad de Castilla-La Mancha, E-13071 Ciudad Real, Spain; \\ victor.ruiz@uclm.es (V.R.-D.); jorge.hernando@uclm.es (J.H.-G.); joseluis.saldavero@uclm.es (J.L.S.-R.) \\ * Corre spondence: victor.ruiz@uclm.es; Tel.:+34-926-295-300
}

\begin{abstract}
This paper reports the design, fabrication and performance of MEMS-based piezoelectric bidirectional convey ors featuring 3D printed legs in bridge resonators. The structures consisted of aluminium-nitride (AIN) piezoelectric film on top of millimetre-sized rectangular thin silicon bridges and two electrode patches. The position and size of the patches were analy tically optimised for the wavegeneration, while the addition of 3D-printed legs, for a controlled contact, allowed for a further step into the manufacturing of efficient linear motors. Such hybrid devices have recently demonstrated the convey ance of sliders - surpassing several times the motor w eight - with speeds of $1.7 \mathrm{~mm} / \mathrm{s}$, while operated at $6 \mathrm{~V}$ and $19.3 \mathrm{kHz}$. How ever, by the optimisation of various aspects of the device such as the vibrational modes and excitation signals speeds above $25 \mathrm{~mm} / \mathrm{s}$ were demonstrated.
\end{abstract}

Keywords: travelling wave; standing wave; bidirectional linear motion; conveyor; piezoelectric; AlN; MEMS

\section{Introduction}

The miniaturization of actuators for applications that need large displacements, high energy efficiency or output forces is an ongoing challenge [1]. Piezoelectric ultrasonic motors (USM) have proven to be a suitable solution to obtain long motion range, high torque, quick response, high pow er to w eight ratio and high efficiency in comparison to electrostatic, magnetic, and thermal alternatives [2-4]. Despite the advantages of USM for linear motion, scaling dow $n$ to the millimetre range remains a challenge, due to the difficulties in generating standing or travelling $w$ aves at high frequencies with enough amplitude [5].

In pursuit of miniaturization, the monolithic fabrication based on silicon micromachining was successfully applied to the effective size reduction of such positional devices. Different actuation techniques have benefited from the MEMS technology, demonstratinglocomotion or conveyancein the millimetre scale with speeds of a few millimetres per seconds and payloads below $50 \mathrm{mg}$ [6-8]. How ever, to reach a step further into the miniaturisation of efficient motors, piezoelectric actuation becomes a promising alternative $[\mathbf{9 , 1 0}$.

In this work, we present a hybrid design for linear locomotion based on piezoelectric MEMS resonators with 3D-printed legs. The MEMS-based resonator consists of a conductive silicon bridge, actuated thanks to an integrated AlN piezoelectric film, sandwiched betw een the silicon film and the metallic electrodes. Analy tical approaches were used to calculate the optimal electrode layout for the efficient generation of the mechanical waves, i.e. standing-w ave or travelling-wave, in the motor. The movement of the surface of the silicon plate $w$ as transferred to a slider through an array of 3D-printed legs attached to its surface, allowing a controlled contact between stator and slider and the amplification of the elliptical or diagonal movement caused by the TW or the SW, respectively. The conveyance of light sliders was demonstrated, and the kinetic capabilities of the motor were characterised under both operation modes.

\section{Device design}


Here we focus on the linear motors based on piezoelectrically actuated bridges with a length $L=10 \mathrm{~mm}$ and a width $W=2 \mathrm{~mm}$. The structure consisted of a silicon substrate with a thickness $t_{s}=30 \mu \mathrm{m}$, an aluminium-nitride (AlN) piezoelectric film with a thickness $t_{p}=1 \mu \mathrm{m}$ and two $500 \mathrm{~nm}$ thick Gold ( $\mathrm{Au}$ ) electrode patches on top, that were neglected in the mechanical analysis. Figure 1 a depicts the layout.
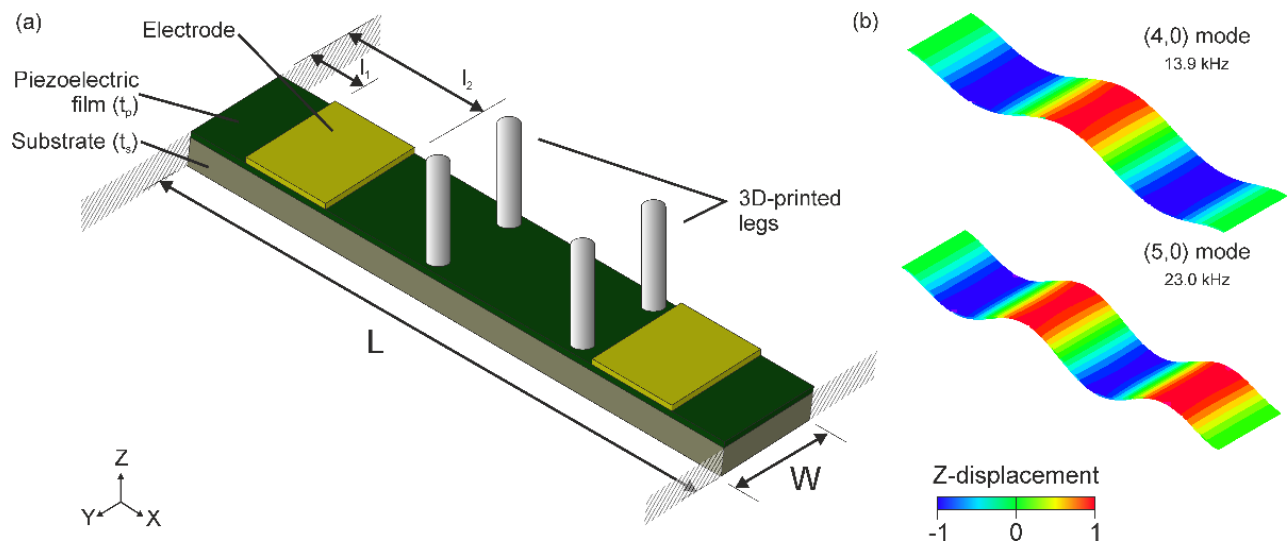

Figure 1. (a) Schematic diagram of the device design. A bridge structure of length $\mathrm{L}$ and width $\mathrm{W}$ consisting of a silicon substrate with a thickness $t_{s^{\prime}}$ covered by an AlN pie zoelectric layer of thickness $t_{p}$. Two symmetrically disposed metallic electrodes we re placed close to the edges, starting at a distance $l_{1}$ and ending at a distance $l_{2}$.3D-printed resin legs we re manually attached in a subsequent step. (b) Mode shapes involved in the motor design. Resonant frequencies a re also included. Colour bar represents the normalised modal dis placement in the Z-axis.

The generation of bidirectional linear motion would rely on the flexural vibration modes of the bridge structure in Figure 1a. In particular, the third and fourth-order bending modes, i.e. $(4,0)$ and $(5,0)$ modes using Leissa nomenclature [11]. Figure 1b shows the modal shapes and their respective resonant frequencies.

The actuation principle of the motor is based on the generation of mechanical waves - either standing-wave (SW) or travelling-wave (TW) types - on the piezoelectrically actuated structure, together with the inclusion of 3D-printed millimetre long legs. These legs will ensure the contact between the vibrating structure and the stator while transmitting a translational motion to the slider.

The generation of linear TW in beams by the combination of two flexural vibration modes was already reported, using two symmetrically placed piezoelectric patches [10]. Here we followed the same approach, obtaining the patch position and size that optimises the TW quality and amplitude with the targeted modes in Figure $1 b$. Regarding the leg position, those should be placed where the TW envelope is ideally constant, to ensure an elliptical trajectory at the tip [12]. That condition is met at the central plateau of the TW envelope, the area between the two patches [10].

Linear motion can also be obtained by inducing an SW in the resonator and choosing an appropriate distribution of legs [13]. A stiff leg placed between a node and a peak of the modal shape wave (Figure $1 b$ ) w ould describe a diagonal trajectory. Depending on the chosen side of the crest, the thrust exerted to the rotor w ould be in the positive or negative direction of the w avelength. Therefore, to achieve bidirectional motion, two different flexural modes can be used. By placing the legs at any of the intersecting regions between each mode chosen side crests, the same device could be used as an SW motor, with two different directions of movement depending on the vibration mode excited.

According to reference [10], the optimal patches for the TW generation could be suitable for the SW generation too. Besides, in order to maximize the excitation of the SW and taking advantage of the two-patch configuration, a phase optimisation was performed [14]. Finally, the combination of this patch design together with attached legs in the intersecting crests regions (SW operation mode) within the central plateau (TW operation mode), w ould allow the same motor to be operated under SW or TW conditions.

\section{Materials and methods}


Monolithic microfabrication techniques were used to implement rectangular microplates according to the geometry and materials previously described, clamped at both sides. For all the devices, the layer structure was as follows: a $30 \mu \mathrm{m}$ thick, p-doped (100), silicon plate served as the bottom electrode, which was covered with a $1 \mu \mathrm{m}$ thick AlN piezoelectric film. As the top electrode, a $500 \mathrm{~nm}$ thick Au electrodes were deposited. Dices with two different devices were glued and wirebonded to a printed circuit board (PCB) to facilitate the electrical access (Figure 2).

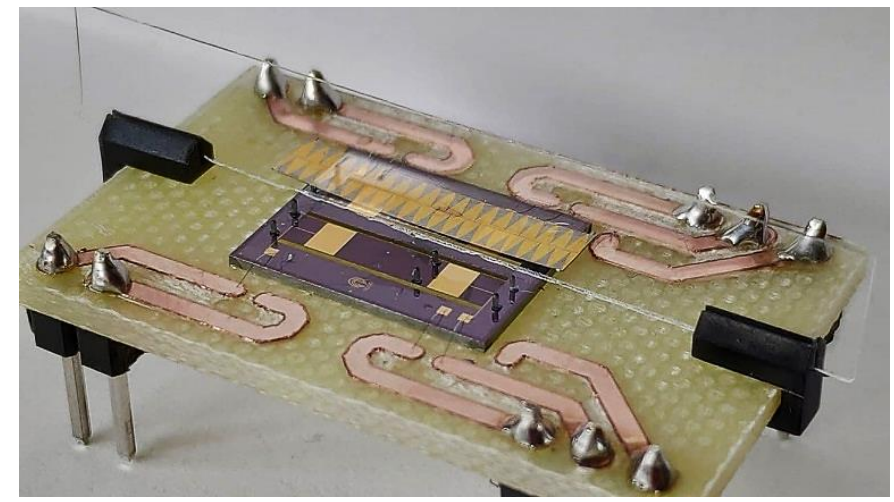

Figure 2. Photograph of the fabricated motor with attached legs. A silicon dice containing two designs was wire bonded to a PCB. In the experimental setup, a gold-patterned slider was placed on top of the motor and constrained to a lane by a vertical glass slice.

Cylindrical legs with a length of $750 \mu \mathrm{m}$ and a diameter of $300 \mu \mathrm{m}$ were designed and manufactured using a B9 Core 530 DLP 3D printer, using proprietary Black Resin. The active pair of legs was glued on the bridge surface using a cyanoacrylate-based adhesive (see Figure 2) and two additional pairs were placed at the device frame, serving as passivesupports.

In the kinetic characterization, a Tektronix AFG 3000 series arbitrary waveform generator (AWG) was used to generate the required waveforms to be applied to each of the electrode patches. In the experimental setup, the PCB containing a pair of such devices (see Figure 2) was placed on a levelled platform - in order to avoid an uneven movement of the slider - under the microscope camera. The orthogonal movement of the sliders was restricted to a rectilinear lane with the help of glass pieces. The movement of the slider was optically recorded by a microscope camera and the videos were processed by a motion tracking algorithm programmed in Matlab, in order to obtain the slider positions versus time. The mean speed was computed afterwards.

\section{Results}

The application of the motor to transport objects in contact with the device surface was assessed in the two operation modes, with sliders consisting of silicon plates of $15 \times 3 \times 0.02 \mathrm{~mm}^{3} \mathrm{with}$ a mass of $2 \mathrm{mg}$. In the TW operation mode, the motor was actuated at $19.55 \mathrm{kHz}$ with $90^{\circ}$ for the forward direction and $-90^{\circ}$ for the reverse direction. In the SW operation mode, the motor was actuated in the $(4,0)$ mode at $15.25 \mathrm{kHz}$ and no phase difference between patches for the reverse direction, and in the $(5,0)$ mode at $24 \mathrm{kHz}$ and a phase difference of $180^{\circ}$ for the forw ard direction.

Figure 3 shows the results from the kinetic characterization in the forward direction (similar results were obtained in the reverse direction). As it can be seen, in the TW operation mode, the motor w as able to develop conveyor speeds of $1.2 \mathrm{~mm} / \mathrm{s}$ at $10 \mathrm{~V}$, while in the SW operation mode, the speed reached a maximum value of $27 \mathrm{~mm} / \mathrm{s}$. This speed of almost $3 \mathrm{BL} / \mathrm{s}$ (body-lengths per second) supposes a huge improvement, comparing to the TW operation mode, mainly due to the actuation of the motor in resonance.

Besides, the minimum excitation to generate locomotion of the slider $w$ as as low as $2 \mathrm{~V}$ in the SW operation mode, with a linear dependence of the speed with the applied voltage up to $4 \mathrm{~V}$, when a speed of $20 \mathrm{~mm} / \mathrm{s}$ was reached. From them, a decrease in the grow th rate of the speed with voltage w as found, what can be attributed to the low inertia of the light slider used in the tests. 


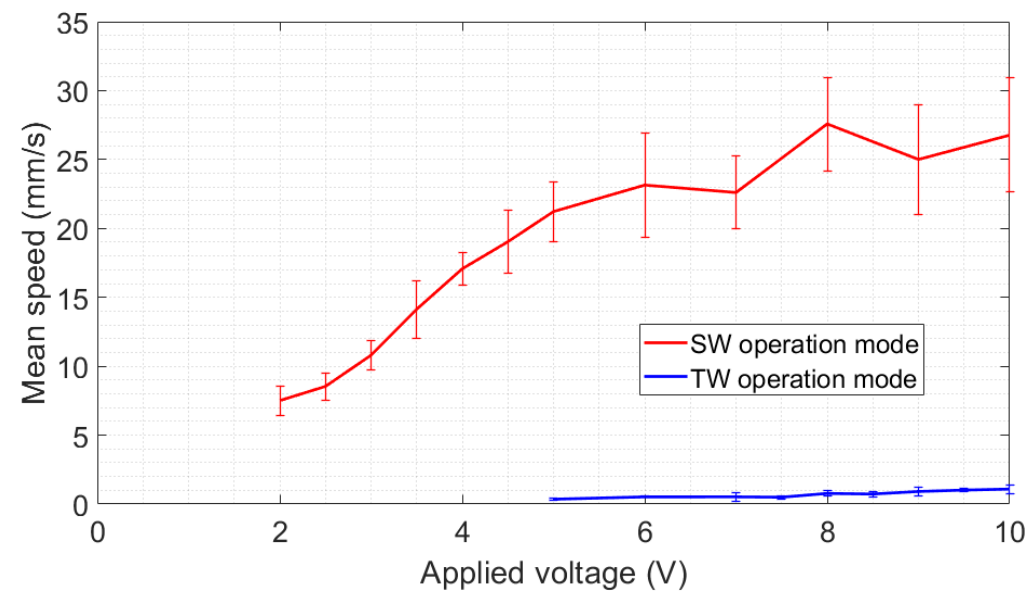

Figure 3. Results from the kine tic characterization of the motor with TW operation mode at $19.55 \mathrm{kHz}$ with a phase difference of $90^{\circ}$ and SW operation mode with the $(5,0)$ mode at $24 \mathrm{kHz}$ and a phase difference of $180^{\circ}$.

\section{Conclusions}

This work reports the design, fabrication and electrical and optical characterization of piezoelectric MEMS plates for linear motion applications. Successful generation of bidirectional TW w as demonstrated on a monolithic microfabricated silicon-based bridge, combined with 3D printed legs, to overcome the intrinsic limitation of the suspended bridge to attain an efficient contact with objects. The bridge devices were also excited in resonance, inducing SW based on the third and fourth flexural modes. The 3D printed legs were placed at those positions in what the resonator could act as a bidirectional resonant motor.

The kinetic performance of the fabricated motor was studied with a $2 \mathrm{mg}$ slider. The conveyor demonstrated bidirectional speeds of $1.2 \mathrm{~mm} / \mathrm{s}$ when a TW w as induced in the resonator at $10 \mathrm{~V}$ and a frequency of $19.55 \mathrm{kHz}$. The same device could be operated in resonance, by inducing SWs based on the $(4,0)$ and $(5,0)$ modes at 15.25 and $24 \mathrm{kHz}$. In this operation mode, the conveyor demonstrated speeds as high as $27 \mathrm{~mm} / \mathrm{s}$ for continuous sinusoidal excitation.

Author Contributions: Conceptualization, J.L.S.-R.; software, V.R.-D.; investigation, V.R-D.; writing - original draft preparation, V.R.-D.; writing - review and editing, J.L.S-R., J.H.-G., H.S. and A.A.; supervision, J.L.S.R. and J.H.-G.; project administration, J.L.S.-R. and J.H.-G.; funding acquisition, J.L.S.-R. and J.H.-G. All authors have read and agreed to the published version of the manuscript.

Acknowledgements: This work was supported by the European Regional Development Fund, the Spanish Ministerio de Ciencia, Innovación y Tecnología project (RTI2018-094960-B-100), and the regional government (JCCLM) project (SBPLY/17/180501/000139).

Conflicts of Interest: The authors declare no conflict of interest.

\section{References}

1. Kenji Uchino MicroMechatronics, Second Edition;

2. Chan, M.L.; Yoxall, B.; Park, H.; Kang, Z.; Izy umin, I.; Chou, J.; Megens, M.M.; Wu, M.C.; Boser, B.E.; Horsley, D.A. Design and characterization of MEMS mic romotor supported on low friction liquid bearing. Sens. Actuators Phys. 2012, 177,1-9, doi:10.1016/j.sna.2011.08.003.

3. Khiat, A.; Spronck, J.W.; van Schieveen, J.; Milosavljevic, S.; Wei, J.; Estevez, P.; Sarro, P.M.; Staufer, U. Linear and rotational thermal micro-stepper motors. Microelectron. Eng. 2012,98,497-501, doi:10.1016/j.mee.2012.07.086.

4. Sarajlic, E.; Yamahata, C.; Cordero, M.; Fujita, H. Three-Phase Electro static Rotary Stepper Micromotor With a Flexural Pivot Bearing. J. Microelectromechanical Syst. 2010, 19, 338-349, doi:10.1109/JMEMS.2010.2040139. 
5. Pulskamp, J.S.; Polcawich, R.G.; Rudy, R.Q.; Bedair, S.S.;Proie, R.M.; Iv anov, T.; S mith, G.L. Piezoelectric PZT MEMS technologies for small-sc ale robotics and RF applic ations. MRS Bull. 2012, 37, 1062-1070, doi:10.1557/mrs.2012.269.

6. Kladitis, P.E.; Bright, V.M. Prototy pe mic rorobots for mic ro-positioning and mic ro-unmanned vehicles. Sens. Actua tors Phys. 2000, 80, 132-137, doi:10.1016/S0924-4247(99)00258-7.

7. Ebefors, T.; Mattsson, J.U.; Kälvesten, E.; Stemme, G. A walking silicon micro-robot. In Proceedings of the in Proceedings of the 10th International Conference on Solid-S tate Sensors and Actuators (Transducers'99; 1999; pp. 1202 1205.

8. Yahiaoui, R.; Zeggari, R.; Malapert, J.; Manceau, J.-F. A MEMS-based pneumatic micro-conveyor for planar micromanipulation. Mechatronics 2012, 22, 515-521, doi:10.1016/j.mechatronics.2011.04.005.

9. Smith, G.L.; Rudy, R.Q.; Polcawich, R.G.; DeVoe, D.L. Integ rated thin-film piezoelectric traveling wave ultrasonic motors. Sens. Actuators Phys. 2012, 188, 305-311, doi:10.1016/j.sna.2011.12.029.

10. Ruiz-Díez, V.; Hernando-García, J.; Toledo, J.; Ababneh, A.; Seidel, H.; Sánchez-Rojas, J.L. Bidirectional Linear Motion by Travelling Waves on Legged Piezoelectric Microfabricated Plates. Micromachines 2020, 11, 517, doi:10.3390/mi11050517.

11. Leissa, A.W.;Qatu, M.S. Vibration of Continuous Systems; McGraw Hill Professional, 2011; ISBN 9780071714808.

12. Hernando-García, J.; García-Caraballo, J.L.; Ruiz-Díez, V.; Sánchez-Rojas, J.L. Motion of a Legged Bidirectional Miniature Piezoelectric Robot Based on Traveling Wave Generation. Micromachines 2020, 11, 321, doi:10.3390/mi11030321.

13. Siyuan He; Weishan Chen; Xie Tao; Zaili Chen Standing wave bi-directional linearly moving ultrasonic motor. IEEE Trans. Ultrason. Ferroelectr. Freq. Control 1998, 45, 1133-1139, doi:10.1109/58.726435.

14. Ruiz-Díez, V.; Manzaneque, T.; Hernando-García, J.; Ababneh, A.; Kucera, M.; Schmid, U.; Seidel, H.; Sánchez-Rojas, J.L. Design and characterization of AlN-based in-plane microplate resonato rs. J. Micromechanics Microengineering 2013, 23, 074003, doi:10.1088/0960-1317/23/7/074003.

(C) 2020 by the authors; licensee MDPI, Basel, Switzerland. This article is an open access article dis tributed under the terms and conditions of the Creative Commons by Attribution (CC-BY) license (http://creativecommons.org/licenses/by/4.0/). 